PharmacoEconomics \& Outcomes News 864, p1 - 17 Oct 2020

\title{
HTA assessment of COVID-19 interventions in Ireland
}

A robust health technology assessment (HTA) system is required for the assessment of COVID-19 interventions during the pandemic, says the National Centre for Pharmacoeconomics (NCPE) Review Group, in an article published in Value in Health.

The NCPE is the national HTA agency in Ireland which assesses the cost effectiveness and budget impact of new medicines submitted by drug companies (applicants) for reimbursement by the state health payer.

Some trials being performed during the COVI-19 pandemic have been temporarily halted or undergone protocol changes, some healthcare services may have been unavailable to patients, and subjects in one treatment arm may have been at increased risk of exposure to COVID-19, resulting in limitations in data leading to uncertainty in HTA assessments that may affect the willingness-to-pay (WTP) threshold and the availability of new treatments. Methods used to assess or correct biases and uncertainties in trial data when evaluating the safety and efficacy of COVID-19 treatments may not be appropriate for cost-effectiveness and budget impact models, said the NCPE Review Group.

Recommendations for applicants making submissions to the NCPE include: general issues (clearly document protocol deviations or statistical analysis changes due to COVID-19; present methods and results with missing data under a range of assumptions; emphasise any structural uncertainty within the model), treatment effect/extrapolation (include results of pre-COVID-19 data as well as latest data in scenario analysis; use sensitivity and scenario analyses to account for COVID-19 impact), evidence synthesis (perform sensitivity analysis to exclude COVID-affected trials if other trials with similar quality are available; investigate potential modifiers and differences if trial data are available before and during the pandemic), QOL (provide scenario analyses on data sources not affected by COVID-19; include a covariate for COVID-19 in analysis of EQ-5D* data), resource use/treatment discontinuation (provide scenario analyses using non-affected data sources), and system change/resource availability (consult updated post-pandemic cost sources; model impact of delays in diagnosis and treatment initiation on patient numbers, costs and outcomes; provide scenario analyses that assume constrained resources; use varying WTP thresholds because the threshold may be reduced under fiscal constraints).

"Trials conducted during the pandemic will be subject to a higher degree of uncertainty than before. It is vital that applicants clearly identify any parameters that may be affected by the pandemic. These parameters will require considerably more scenario and sensitivity analyses to account for this increase in uncertainty," said the NCPE Review Group.

* EuroQol five-dimensions

Leahy J, et al. Coronavirus Disease 2019: Considerations for Health Technology Assessment From the National Centre for Pharmacoeconomics Review Group. Value in Health : 5 Oct 2020. Available from: URL: https://doi.org/10.1016/j.jval.2020.09.003 